\title{
Knowledge Management e modelli strategici in sanità. Una review strutturata della letteratura
}

\begin{abstract}
Le aziende sanitarie stanno fronteggiando un tema fondamentale: erogare servizi quantitativamente $e$ qualitativamente sempre migliori a fronte di risorse decrescenti. Gli studi di Public Management hanno da tempo visto nella generazione di valore pubblico una possibile soluzione a questo problema, ma recentemente hanno contribuito a questo dibattito anche gli studiosi di Business Model nell'intento di fondere assieme elementi di teoria e di pratica. A tal scopo, ben si addicono i risultati delle ricerche sul Knowledge Management, che stanno progressivamente ridisegnando i modi di concepire la produzione e offrire i servizi. Questo paper si colloca in tale contesto per verificare, attraverso una revisione strutturata della letteratura, l'esistenza e la disseminazione di framework ad hoc per le aziende sanitare, che possano risultare utili nelle scelte strategiche delle aziende sanitarie. I risultati della ricerca mostrano che molto rimane ancora da fare dal momento che gli studi oggi disponibili consistono perlopiù in adozioni acritiche di modelli sviluppati per le imprese private. In conclusione sono dunque fornite delle linee-guida per le ricerche future.

Parole chiave: gestione della conoscenza, modelli strategici, aziende sanitarie, crisi economica, revisione della letteratura, Valore pubblico
\end{abstract}

Title: Knowledge Management and new Business Models in Healthcare Organization. The contribution of a SLR (Structured Literature Review)

Abstract. Healthcare organizations face a major challenge: delivering more and better public services, while facing fiscal stress, in other term generating public value. Public value has been central in public management research, but recently also Business Model Design scholars have analyzed how healthcare organizations can create value. In order to sharoen research and practice, business model research can fruitfully be combined with knowledge management research. The evolution of KM practices, in fact, is reshaping the traditional way the organizations operate. In this light, the present paper aims at verifying the existence of ad hoc frameworks for public healthcare organizations and their dissemination through a structured literature review. The research shows that a lot has still to be done since the available studies generally consist in uncritical applications of models designed for private companies. The paper also provides some guidelines for future research.

Keywords: Healthcare Knowledge Management, Business Model, Fiscal Stress, Public Value, Public Management, Structured Literature Review

\section{Introduzione}

La consistenza delle risorse pubbliche impiegate nel settore sanitario, la recente crisi economica e i connessi tagli alla spesa pubblica, l'invecchiamento media della popolazione (Snowdon and Chen, 2011; Thim and Weber, 2012; Levy e Sobolev, 2016; http://www.hspm.org/mainpage.aspx, ultima consultazione 17 marzo 2018) pongono con forza il tema del recupero di competitività delle aziende sanitarie, pubbliche e privateconvenzionate.

Nelle aziende pubbliche è stata a lungo studiata l'efficienza nei processi di acquisizione e di impiego delle risorse; viceversa, sono stati analizzati meno in profondità i fattori strutturali e le dinamiche di cessione dei servizi. Con riguardo alla sanità ci si riferisce alle caratteristiche della domanda di salute, alla differenziazione dell'offerta dei servizi e ai connessi valori economici generati (Longo e Ricci, 2017). La ragione di fondo è probabilmente da ricercarsi nel contesto di riferimento che nel caso di specie è un "quasi-mercato" nel quale i principali clienti sono rappresentati da un numero limitato di potenziali attori, coincidenti in larga massima con le istituzioni regionali (espressione dei cittadini) e, secondariamente, con i fondi sanitari e pazienti solventi. $\mathrm{Da}$ ciò la sottovalutazione delle strategie competitive relative all'innovazione di prodotto, di mercato e, conseguentemente, di valore aziendale. A indebolire ulteriormente l'attenzione su questo profilo va poi aggiunto che, nella maggior parte dei casi, chi fruisce dei servizi (i pazienti) non corrisponde con chi paga (regioni e fondi sanitari privati) ma anche che il controvalore delle prestazioni è rappresentato da tariffe pubbliche e non da prezzi di mercato (Aa.Vv., 2012).

Tali caratteristiche rendono senz'altro complesso ragionare in termini di valore aziendale, al pari di quanto accade per gli altri settori della pubblica amministrazione e a differenza dei contesti privati. L'assenza di un mercato tout court, in particolare, rende difficoltosa non tanto la correlazione dei processi di acquisizione e impiego dei fattori della produzione a quelli di cessione dei servizi, quanto l'interpretazione dei risultati di sintesi come espressi, ad esempio, dal reddito di esercizio o dal patrimonio netto. Si tratta tuttavia di valutazioni 
possibili, a patto di utilizzare le giuste chiavi interpretative e di tenere in opportuna considerazione il finalismo proprio delle aziende sanitarie e il relativo contesto di riferimento che è caratterizzato, lo si è anticipato, dalla presenza di pochi grossi clienti, distinti dai fruitori, da intense relazioni inter-istituzionali e da tariffe pubbliche che sostituiscono i prezzi di mercato.

Si tratta, a ben vedere, di un accogliere un approccio olistico nei confronti dell'azienda e di intendere i processi aziendali tra loro interconnessi ed in continua relazione con le finalità proprie degli istituti (di cui le aziende rappresentano una mera astrazione economica). Dunque, in termini operativi, di avvalersi di misure extracontabili a complemento delle condizioni di equilibrio economico. Prospettiva, questa, maturata dagli studiosi italiani sin dai primi contributi di Gino Zappa e propria del pilastro dell'economicità come posta da Carlo Masini e successivamente sviluppata da Airoldi, Brunetti e Coda. Scrive in particolare Masini: "l'indagine intorno al soddisfacimento dei fini istituzionali trova dati molteplici nel sistema di quantità economiche anche nella espressione di sintesi di valori; ma per il contemperamento tra il soddisfacimento dei fini primi definiti per i bisogni comunitari ... e i fini congiunti necessariamente di rimunerazione di coloro che prestano lavoro nell 'istituto, sono indispensabili informazioni in quantità monetarie e non monetarie di varia specie, spesso di difficile apprezzamento globale” (Masini, 1970).

\section{Il Background di riferimento}

Dal primo contributo di Moore (1995), il tema della competitività è stato ampiamente dibattuto sia dagli studiosi di Public Value che dai Policy Maker (Williams and Shearer, 2011) ${ }^{1}$. L'idea centrale di questo approccio (Moore, 1995; 2013) consiste nel considerare le risorse pubbliche, in particolare quelle umane, uno strumento di creazione del valore. Moore parla in particolare di un "triangolo strategico" che comprende tre fattori reciprocamente interdipendenti: (1) una proposta di valore pubblico consistente in una dettagliata dichiarazione di intenti che contempli i bisogni della comunità di riferimento e la loro compatibilità con le sottese metriche di rendimento (sostenibilità); (2) la raccolta di consenso mediante il coinvolgimento di tutti gli stakeholder rilevanti e non solo della rappresentanza politica eletta (Donahue e Moore, 2012); (3) l'effettiva conduzione aziendale secondo la proposta di valore condivisa.

Si tratta di un inquadramento teorico di notevole attualità che possiede un interessante potenziale empirico, sul quale tuttavia ancora oggi rimangono aree di opacità, sia dal lato della teoria (cosa è in concreto il valore pubblico? Come si misura?) che da quello pratico di pubic policy e conduzione aziendale. Non è un caso, d'altronde, che il dibattito sul significato di Public Value, delle sue applicazioni e delle sue implicazioni è tuttora vivace e coinvolge studiosi di più discipline (Alford et al., 2016).

Tra gli altri, il tema del valore pubblico ha recentemente attratto l'attenzione degli studiosi di strategia (Kaplan, 2011), anche grazie al coevo sviluppo dei paradigmi di Knowledge Management (d'ora innanzi anche KM). La ragione sta con ogni probabilità nella complementarità dei due approcci: tra gli studi di strategia, infatti, quelli incentrati sui modelli di Business intendono fornire alle aziende gli strumenti e le modalità per catturare, creare e trasferire valore, indipendentemente dal loro finalismo ultimo (profit o no profit); il KM, dall'altra parte, indaga le modalità attraverso cui la conoscenza può migliorare i processi organizzativi, fornire gli strumenti per produrre beni o erogare servizi e, conseguentemente, trasformare le connesse funzioni aziendali. La sintesi tra Business Model e KM è pertanto facilmente realizzabile e il potenziale è di grande rilievo. Si pensi solo che le pratiche di gestione della conoscenza, se associate alle potenzialità offerte dallo sviluppo della tecnologia e degli strumenti informatici, permettono già oggi di superare gli annosi problemi legati alla prossimità delle strutture e ai tempi di attesa, ma anche a quelli dell'efficacia predittiva delle diagnosi e dell'efficacia terapeutica delle cure, come stanno a dimostrare le opportunità aperte dall'impiego dei robot chirurgici, dalla telemedicina (Cegarra-Navarro et al., 2012; Cruz-Cunha et al., 2013) e in generale dalla possibilità di avviare nuove forme collaborazione inter-istituzionale (Mabery et al., 2013).

Nonostante tutto questo, mentre nel settore privato gli studiosi hanno sviluppato nel tempo modelli interpretativi di indiscusso successo, oggetto peraltro di numerosi contributi teorici e di volumi divulgativi, nel settore pubblico non sembrano emergere soluzioni specifiche che non rappresentino meri adattamenti delle soluzioni sviluppate per il settore privato for profit (Garlatti et al., 2015). Da qui l'interesse di chi scrive per

\footnotetext{
${ }^{1}$ Per la verità, come anticipato in introduzione, gli studiosi italiani avevano anticipato il tema già dagli anni ' 70 dello scorso secolo mediante lo sviluppo del concetto di economicità e successivamente attraverso un'interessante apertura al dibattito internazionale (Borgonovi e Meneguzzo, 1990).
} 
l'approfondimento di questo specifico argomento attraverso la conduzione di una revisione strutturata della letteratura internazionale in materia di sanità.

\section{Obiettivo e ragioni del contributo}

Questo lavoro intende verificare se la teoria e la prassi hanno sviluppato e impiegano modelli di business capaci di interpretare l'attuale contesto socio-economico, connotato dalla riduzione delle risorse, da una parte, e dal costante aumento quali-quantitativo di domanda di salute dall'altra. Più in dettaglio ci si chiede quale contributo hanno fornito in tal senso gli studi di Knowledge Management. Il tutto senza entrare nel merito delle possibili modifiche dei modelli sanitari (che sono possibili ma che non sono trattati in questa sede), quindi a sistema invariato, verificando dunque la possibilità di superare l'apparente contraddizione della scarsità di risorse combinate alla domanda crescente di prestazioni. L'analisi si sofferma sui contributi di KM in ambito sanitario (d'ora innanzi anche Healthcare Knowledge Management), presentando i risultati di una revisione sistematica della letteratura (cfr., infra, paragrafo 4, per le ipotesi di base adottate, gli strumenti di ricerca utilizzati e i limiti connessi dell'analisi).

Numerose sono le ragioni che motivano questo studio e la prospettiva adottata, quella come detto del KM.

Già si è accennato alle dinamiche socio-economico di carattere generale (invecchiamento della popolazione e connesso incremento di prestazioni socio-sanitarie) e alla necessità di contenere la spesa pubblica sanitaria per preservare gli equilibri di finanza pubblica. Ebbene, solo in Italia la spesa sanitaria si aggira intorno ai 115 miliari di euro (http://www.agenas.it/, ultima consultazione 8 aprile 2018) mentre è stato stimato che oltre il $70 \%$ della spesa sanitaria nei Paesi OECD proviene da risorse pubbliche (Varabyova e Schreyögg, 2013). Indipendentemente dal dibattito politico costantemente in atto sulla consistenza della spesa (le opinioni variano ad esempio in base al fatto che la spesa sanitaria sia considerata in termini assoluti o in rapporto al PIL) e della sua adeguatezza in rapporto ai servizi offerti, sta di fatto che in tempi di rientro dalla crisi finanziaria le uscite per la sanità rappresentano un'entità a due cifre della spesa pubblica nel suo complesso (circa il 14\%) e pertanto oggetto di attenzione. È poi un dato di fatto che, all'interno del contesto europeo, l'età media della popolazione italiana è seconda solo a quella della Germania con un tasso di crescita addirittura doppio rispetto a quella della media degli altri Paesi; il che porta le aspettative di vita alla nascita a 84,6 anni e 80,1 anni rispettivamente per donne e uomini favorendo le richieste di cure per malattie croniche, disabilità e non autosufficienza (http://www.istat.it/it/, ultima consultazione 8 aprile 2018); in breve al "bisogno di salute" e di "domanda sanitaria".

Per queste ed altre ragioni (la necessità di fornire indicazioni di policy garantendo rigore metodologico della ricerca, ad esempio) gli studiosi sono invitati da tempo a comparare i sistemi sanitari (a livello macro), ad analizzare l'efficacia delle policy (a livello meso) e a diffondere le best practices (a livello micro) (Papanicolas e Smith, 2013). Va detto, a tal proposito, che nonostante gli sforzi compiuti negli ultimi anni dai ricercatori e dalle istituzioni, ad oggi non è ancora possibile sostenere la superiorità di un sistema sanitario rispetto ad altri e, conseguentemente, indirizzare le scelte dei governi verso modelli di offerta astrattamente migliori, a meno di non adottare approcci econometrici puri (Lombrano, 2018). Appare invero più utile e pragmatico ricercare l'equilibrio dei sistemi sanitari partendo da quello delle singole parti (aziende) che li compongono.

A tal proposito, la prospettiva di analisi offerta dagli studiosi di Knowledge Management appare particolarmente utile per più ragioni che vanno dalla pluralità di attori coinvolti nell'offerta dei servizi, alla interdipendenza e interdisciplinarità dei relativi bagagli culturali, al rapido sviluppo delle conoscenze e alla conseguente necessità di specializzazione, alla complessità insita nel trasferimento delle conoscenze sanitarie, al considerevole e crescente impiego della componente tecnologica. In effetti le professionalità interne ed esterne alle realtà sanitarie sono varie e specifiche, forse più che altrove, e l'obiettivo consiste nel metterle in relazione; possibilmente a sistema (Bordoloi e Islam, 2011). Oltre a quelle sanitarie, in funzione dei casi, esse includono quelle detenute degli amministratori, dai general e dagli operation manager, dagli informatici e dagli ingegneri clinici, dai fornitori di farmaci, di dispositivi medici e di tecnologia, dagli assicuratori, dalle società scientifiche e dai contraenti (pubblici o privati) di prestazioni. Cruz e Ferreira (2016) sostengono che la conoscenza posseduta da ciascuno di questi attori è fondamentale per l'erogazione di un servizio competitivo ma che la stessa va vista nel suo complesso, individuando le forme di interazione, condivisione e disseminazione delle informazioni (Sabeeh et al. 2016).

Anche volendo circoscrivere il discorso alle sole figure sanitarie, mediche e non, la sfida non cambia. Le conoscenze in sanità sono infatti variegate (cliniche, infermieristiche, sanitarie in generale - Oborn et al., 2013) 
e, specie quelle mediche, sembrano difficilmente trasferibili tramite la loro esplicitazione, come succede per le prassi cliniche o chirurgiche in senso stretto, ad esempio (Sabeeh et al. 2016). In tal senso alcuni autori hanno sottolineato l'importanza di individuare prassi e strumenti ad hoc per condividere le esperienze, suggerendo ad esempio di valorizzare la pratica clinica, da strutturare e mettere a sistema con il bagaglio di conoscenze esplicite o esplicitabili (Yamazaki e Umemoto, 2010). Altri studiosi, riconoscendo la difficile trasferibilità di queste conoscenze, si sono limitati a osservare l'importanza di esplicitazione di chi sa cosa, quali sono le fonti della conoscenza e come vi si può accedere: anche questo diventa una parte importante base di successo (Persson e Stirna, 2007).

L'esplicitazione della conoscenza, peraltro, non deve condurre all'effetto indesiderato della burocratizzazione e della responsabilizzazione formale, che rappresentano un concreto rischio di deriva da cui il KM intende rifuggire (Yamazaki e Umemoto, 2010). L'attribuzione di responsabilità formali genera infatti atteggiamenti tipici di "evasione da responsabilità" e prassi di medicina difensiva (accertamenti diagnostici pletorici e spesso inutili) che vanno in senso opposto alla qualità e alla riduzione dei costi della sanità. Chiari esempi di questo tipo sono rappresentati dalle migliaia di linee guida elaborate dalle società scientifiche (Griffith et al., 2013), in Italia spesso supportate dalle legislazioni nazionali e regionali di settore, tradotte poi in procedure interne e impersonate dalle figure delle direzioni sanitarie. La situazione non è diversa negli altri Paesi: in Inghilterra, ad esempio, ogni anno vengono consegnate ad ogni medico $15 \mathrm{Kg}$ di procedure (Dwivedi et al., 2003); altrove è stata riscontrata la presenza (e vigenza!) di ben 855 diverse linee guida - una pila di 68 centimetri di carta e di 28 chili di peso" (Booth, 2000).

Va infine considerato che la sanità è un settore ad alta intensità e concentrazione di tecnologia, territorio ideale per l'avanzamento della società della conoscenza. Tale convincimento è ampiamente diffuso e trova continue conferme con il passare degli anni: nel 2010 Williams e Dickinson (2010) sostenevano che le tecnologie potessero assumere fisionomie varie, tali da ricomprendere nuove procedure mediche e prodotti farmaceutici, impiego di sistemi di informazione e comunicazione, innovazioni nel management dei servizi. Qualche anno dopo Delgosha et al. (2013) arrivano ad affermare che l'assistenza sanitaria è da considerarsi al pari di un'industria che sperimenta tecniche e approcci progressivamente più innovativi. Ancor più recentemente Sabeeh et al. (2016) hanno constatato la complessità di contesto dei sistemi sanitari, caratterizzato dalla crescita sostanziale delle conoscenze scientifiche, dall'impatto degli errori medici, dagli alti investimenti in tecnologia e dall'implementazione di nuovi modelli gestionali. Terreno fertile e particolarmente adatto, dunque, per l'implementazione della gestione della conoscenza.

In generale è pressoché unanime il convincimento che anche le aziende pubbliche, e non solo quelle che competono sui mercati tradizionali, debbano implementare modelli di business innovativi in grado di supportare l'offerta di nuovi servizi e, conseguentemente, di ri-disegnare i processi aziendali tipici (sanitari, tecnici e amministrativi) ma anche di sviluppare rinnovate modalità di comunicazione inter e intraorganizzative (Macdonald, 2003; Kothari et al., 2011; Obrn et al., 2013). Affinché queste innovazioni possano tradursi in valore aziendale, tuttavia, le risorse offerte dalla tecnologia e dagli strumenti informatici debbono essere sfruttati con pertinenza e gradualità. L'impiego di tali risorse, infatti, deve essere coerente con il contesto di specie, quindi con le caratteristiche dei fruitori dei servizi e con i loro finanziatori, con le relazioni istituzionali e il contesto normativo, con la specificità dei processi organizzativi (sanitari e non) e con i mercati di approvvigionamento; evitando dunque l'adozione acritica di soluzioni sperimentate, spesso con successo, in settori diversi, che in troppi casi non si stanno traducendo nel miglioramento delle performance aziendali.

\section{Quesiti e metodologia di ricerca}

Dato lo specifico oggetto di analisi (il contributo del Knowledge Management nello sviluppo e impiego di modelli innovativi di Business in sanità) ci si è posti le seguenti domande:

(a) in che misura gli studi di Knowledge Management in sanità affrontano specificatamente argomenti di management (innovazione di prodotto, organizzazione e gestione per processi, dinamiche del valore, ad esempio) e in quale altra misura approfondiscono la gestione delle informazioni in ambito clinico, tecnologico o informatico?

(b) Quanti di questi studi sviluppano framework propri, avuto dunque cura delle caratteristiche tipiche delle aziende analizzate (strutture, processi e ambiente economico/istituzionale di riferimento)? I framework sono stati testati?

(c) Le dimensioni del valore sono opportunamente esplicitate? In particolare: sono individuati i driver del valore? Come è misurata la creazione di valore e come è correlata ai suoi driver? 
La ricerca è stata condotta seguendo il consolidato metodo delle revisioni sistematiche della letteratura per come recentemente adattato agli studi di management (Massaro et al. 2016). Il lavoro ha visto tre fasi: (1) l'individuazione dei contributi di Healthcare Knowledge Management, (2) la definizione di quelli astrattamente interessanti perché relativi all'oggetto di questo articolo, (3) la selezione e l'analisi di quelli effettivamente pertinenti.

Per individuare i contributi di Knowledge Management specificatamente rivolti a indagare le performance in sanità ci si è avvalsi di Scopus e Web of Science (WoS), che sono considerati i principali database di riferimento per le scienze sociali. Gli algoritmi di ricerca sono stati impostati in modo ampio, nell'intento di catturare il maggior numero possibile di saggi scientifici pertinenti con l'oggetto di studio e riservare la selezione di quelli rilevanti alla lettura degli abstract; per lo stesso motivo non sono state inserite limitazioni di data. Per Scopus è stato usata la stringa "Titolo: Knowledge Management AND Titolo/KW/Abstract: Healthcare"; per WoS "Titolo: Knowledge Management AND Topic: Healthcare". Sono quindi stati eliminati i titoli doppi, fattispecie ampiamente ricorrente a causa della presenza degli stessi paper su entrambi di DB consultati, della pubblicazione di alcuni lavori su più riviste o apparsi prima in atti di convegno e poi su rivista (in questi casi sono stati mantenuti, rispettivamente, quello più recente e quello apparso su rivista) o della presenza di errori materiali di caricamento delle informazioni nei database. I contributi rintracciati sono stati 685 in totale (dei quali 439 su Scopus e 246 su WoS), divenuti 466 dopo l'eliminazione di 219 titoli doppi.

Sono quindi stati letti i titoli, le parole chiave e, nei casi dubbi, gli abstract dei lavori selezionati per verificare la loro rispondenza a pre-determinati criteri di inclusione nella revisione. Nel dettaglio non sono stati scelti i lavori che, pur ricomprendendo nel titolo le parole Knowledge e Management, alternativamente non facevano riferimento alla gestione della conoscenza o non erano incentrati sulla sanità. Sono stati invece mantenuti i paper che, pur non inquadrabili nei paradigmi del KM in senso stretto, sembravano affrontare in generale i temi del management della conoscenza in sanità. Da ultimo sono stati eliminati i titoli degli atti di convegno dal momento che i singoli contributi che li compongono compaiono autonomamente nei database. Dei 466 saggi rintracciati nella prima fase della ricerca, ne sono rimasti 358 potenzialmente interessanti per il seguito dell'analisi.

Si è dunque passati alla lettura estensiva degli abstract per selezionare le ricerche più attinenti all'oggetto specifico di questa analisi. Al fine di includere il maggior numero di informazioni possibile non si è badato alla prospettiva di analisi adottata dagli autori (macro, meso o micro), come pure non è stata effettuata alcuna selezione in base ai disegni, ai metodi e agli strumenti di ricerca utilizzati, né si è distinto in base allo specifico oggetto indagato (includendo dunque i sistemi sanitari e le relative articolazioni, le aziende sanitarie e le aziende ospedaliere pubbliche comunque denominate, le strutture sanitarie private convenzionate con il sistemi sanitari) o all'area geografica dei casi di studio analizzati. Sono invece stati scartati, in quanto non oggetto precipuo di interesse, i paper relativi a imprese fornitrici di tecnologia, farmaci, dispositivi medici e sanitari (a meno di analisi focalizzate sul rapporto con le aziende del sistema sanitario), nonché i contributi inerenti la gestione delle informazioni e delle conoscenze in ambito propriamente clinico o tecnico-informatico privi di riferimenti ai driver del valore. Sono rimaste 112 ricerche nel complesso, delle quali 18 sono parse di particolare interesse per l'autorevolezza della fonte della pubblicazione o la pertinenza con le dinamiche del valore.

Tab 1 - Numerosità dei contributi individuati e selezionati

\begin{tabular}{|c|c|c|}
\hline & Scopus & Web of Science \\
\hline Totale articoli individuati & 439 & 246 \\
\hline di cui doppi & \multicolumn{2}{|c|}{219} \\
\hline Articoli considerati in totale (primo step) & \multicolumn{2}{|c|}{466} \\
\hline Non rientranti nei criteri di inclusione & \multicolumn{2}{|c|}{108} \\
\hline Articoli potenzialmente interessanti (secondo step) & \multicolumn{2}{|c|}{358} \\
\hline Articoli esclusi dopo lettura estensiva degli abstract & \multicolumn{2}{|c|}{246} \\
\hline Articoli selezionati (terzo step) & \multicolumn{2}{|c|}{112} \\
\hline di cui di particolare interesse per contenuto & \multicolumn{2}{|c|}{18} \\
\hline
\end{tabular}

Per rispondere alle domande poste in apertura di paragrafo ci si è avvalsi di un framework concettuale sviluppato ad hoc, che ha permesso di codificare i contributi in base alle seguenti variabili:

- elementi di innovatività; vengono qui esplicitati i risultati della ricerca condotta. Ne sono possibili esempi: (1) lo sviluppo di specifici framework o l'adattamento di framework esistenti al contesto analizzato; (2) la conferma/smentita di framework esistenti (o di singole ipotesi) mediante analisi empiriche; 
- variabili analizzate (ad esempio creazione di conoscenza, forme e strumenti di esplicitazione della conoscenza, forme di cooperazione, leadership, barriere verso l'innovazione) e presenza di una loro correlazione esplicita con le performance aziendali;

- metodologia di ricerca utilizzata. Tra le numerose classificazioni disponibili si è scelto di utilizzare quella proposta da Cacace et al. (2013) perché recente e supportata da importanti basi epistemologiche. I contributi sono quindi stati selezionati in base a: (1) disegno di ricerca (conceptual paper, revisione della letteratura, singolo caso di studio, pochi casi - sino a 10, numerosi casi, sopra i 10), (2) strategia comparativa, se e ove pertinente (orientata ai casi di studio o alle variabili) e (3) metodologia generale (qualitativa, quantitativa o mista).

La ricerca possiede sostanzialmente due limiti. Il primo è insito nell'individuazione dei database consultati: Scopus e Web of Science. La scelta poteva ricadere su altri fonti, come ad esempio Medline, Cinahl e Health Source: nursing/academic edition, certamente più centrate sulla sanità, ma inevitabilmente più lontane dall'approccio investigativo delle scienze sociali. Il secondo limite consiste nell'assenza di validazione dei risultati di ricerca mediante duplicazione della review da parte di "pari indipendenti", come vorrebbero le più rigorose metodologie. Complessivamente, dunque, il rischio è quello di non aver considerato contributi significativi perché apparsi su riviste non censite dai database consultati ovvero perché scartati a causa di errore (o sensibilità) riconducibile agli autori. Per attenuare questa alea e trovare una fonte autorevole di triangolazione si è comunque interrogato google scholar utilizzando le stesse parole chiave; lo stesso motore di ricerca ha permesso di confermare la solidità della selezione effettuata.

\section{Risultati dell'analisi}

La ricerca condotta ha consentito anzitutto di confermare che il Knowledge Management è un paradigma interdisciplinare (Ferlie et al., 2012). Sono in effetti numerose le discipline che adottano tale approccio; escludendo solo temporaneamente quello manageriale, che sarà discusso in dettaglio, e stando ai lavori individuati nella seconda fase di questa ricerca (cfr., supra, paragrafo 4), possono essere definiti i seguenti cluster logici:

- contributi squisitamente clinici, relativi quindi conoscenza di dati e percorsi utili al trattamento pazienti affetti da specifiche patologie, specie croniche (diabete, osteoporosi o demenza senile, per esempio). Più dettagliatamente, tali studi possono riguardare: (i) dati/percorsi/procedure mediche, (ii) pratiche assistenziali (infermieristiche e di altre professioni sanitarie - di biologi, ostetriche, fisioterapisti, tecnici sanitari etc), (iii) questioni di Risk Management (igiene sanitaria). Esempi tipici sono, nello stesso ordine: (i) impiego di informazioni cliniche atte alla decisione dell'atto medico da intraprendere (diagnostica, cure, interventi invasivi), (ii) movimentazione del paziente, (iii) prevenzione del rischio clinico in struttura (infezioni, in particolare, ma anche cadute, lavaggio mani etc);

- $\quad$ saggi tecnici, a loro volta da distinguersi tra: (i) analisi di funzionalità/efficacia dei prodotti informatici (visti come strumenti di supporto alla conoscenza) e delle loro integrazioni, (ii) progetti e prototipi innovativi quali ad esempio la telemedicina, (iii) studi di semiotica (ed in particolare semantica) relativi agli strumenti informatici di supporto al KM (ex: Resource Description Framework - RDF o Web Ontology Language - OWL), (iv) valutazioni di ingegneria clinica (installazione e gestione di apparecchiature bio-mediche);

- paper incentrati sulla "esplicitazione (comunicazione) della conoscenza" ai pazienti finalizzata al loro diretto coinvolgimento nelle attività di prevenzione, nelle decisioni diagnostiche o nelle cure. Sono esempi di questo tipo la promozione di corretti stili di vita (prevenzione), la divulgazione delle condizioni cliniche o della sintomatologia che suggerisce sottoporsi ad accertamenti (diagnostica) o le buone prassi da adottare in particolari tipi di cure (malati cronici):

- esistono infine ricerche di matrice sociologica (che studiano l'impatto delle innovazioni sui gruppi e sui singoli), di tipo politologico (che osservano il ruolo che può giocare la gestione delle informazioni e della conoscenza sul consenso politico) e quelle relative ai diritti dei cittadini (in particolare alla privacy). 


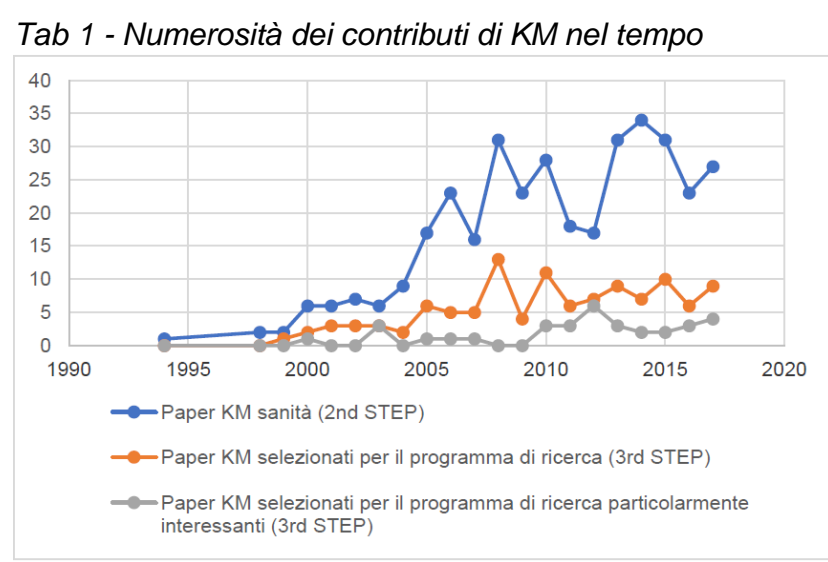

Fonte: ns elaborazione

\section{Tab 1 - Concentrazione dei contributi di KM nel tempo}

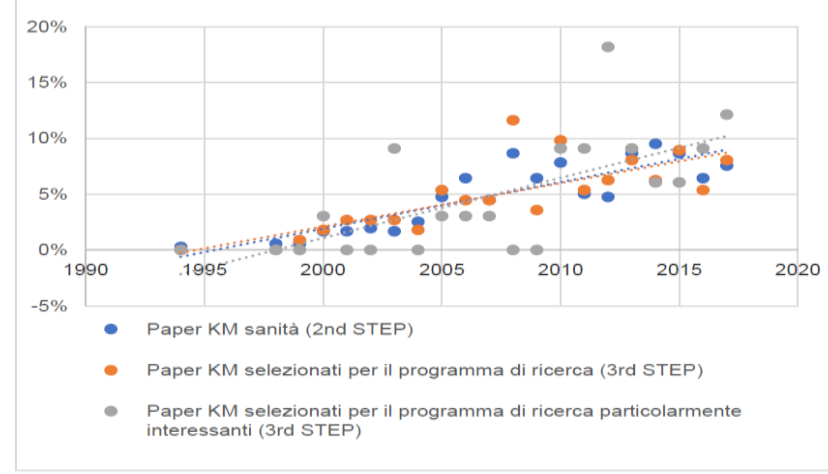

Fonte: ns elaborazione

I contributi aventi ad oggetto i temi di questa ricerca, lo si è visto in precedenza, appaiono invero una minoranza di quelli che trattano in generale di gestione della conoscenza (112 su 358), nonostante la scelta mirata dei database (cfr. paragrafo 4). Sono contributi di management in senso stretto (quindi propri delle discipline economico-aziendali) che comprendono, tra gli altri: (i) l'analisi dei fattori di successo o ostativi la diffusione del $K M$, (iv) la percezione delle iniziative di $K M$ da parte degli operatori e degli Stakeholder in generale, (v) le valutazioni tangibili (risultati) di iniziative di $K M$, (vi) gli strumenti di certificazione ISO e disclosure di $K M$. Come mostrano le figure 1 e 2, la loro distribuzione nel tempo rispecchia quella degli altri studi di Knowledge Management. È possibile in particolare affermare che la loro diffusione avviene intorno agli anni 2000, per crescere sensibilmente tra il 2010 e il 2015 e successivamente raggiungere la maturità. Per motivi di spazio e di interesse ci si sofferma in questa sede sui 18 contributi che sono apparsi all'analisi più significativi. Essi appaiono ordinabili in base al metodo di ricerca adottato e all'area di approfondimento tematica (cfr. tabella 2).

Riguardo alla metodologia impiegata dagli studiosi si può osservare che le revisioni della letteratura e le analisi di esperienze sono largamente impiegate (rispettivamente 7 e 8 paper su 18) mentre le elaborazioni teoriche sono meno ricorrenti (3 contributi). L'approccio di tipo qualitativo è predominante e, oltre ad interessare le revisioni della letteratura, riguarda la maggior parte dei casi studio. Per questi ultimi si contano solo due analisi quantitative (Cruz e Ferreira, 2016; Wu e Hu, 2012) e un'analisi mista (Dwivedi et al., 2003). In generale non si è riscontrata alcuna correlazione tra tematica affrontata (cfr, infra, questo paragrafo) e metodologia d'indagine prescelta, a conferma dell'unitarietà disciplinare dei contributi selezionati per l'analisi e dei cluster individuati.

Rispetto ai contenuti, in linea di massima è possibile individuare tre aree di approfondimento $(c f r$. tabella 2). Una prima linea di ricerca esplora le variabili tipiche degli studi di KM (e relativi framework) per individuare le strategie da adottare nelle aziende sanitarie. Ferlie et al. (2012) propongono una lettura interdisciplinare dei contributi apparsi su 29 riviste dal 2000 al 2008, soffermandosi poi sui temi propri della prospettiva gestionale: (1) tecnologia e sistemi informativi, (2) apprendimento organizzativo, (3) forme organizzative, (4) trasferimento di conoscenza e performance, (5) barriere e facilitatori dello sviluppo organizzativo, (6) cultura e comunicazione, (7) ricerca e sviluppo. Pongono dunque l'accento sull'importanza della conoscenza, intesa come risorsa aziendale sulla quale investire, specie nei contesti di mercato o di quasi mercato. Sono infatti le forme organizzative dei network e delle partnership, più che i mercati e le gerarchie tradizionalmente intese, a favorire lo sviluppo e la condivisione della conoscenza. Sabeeh et al. (2016) vanno oltre, analizzando i punti di debolezza di tre modelli presenti in letteratura ${ }^{2}$ e sviluppandone un quarto, che denominano Healthcare Knowledge Management Framework. Tra le altre, gli Autori pongono l'enfasi su alcune variabili poco esplorate come la percezione del ruolo della conoscenza da parte delle persone, la cultura della sua condivisione, i depositari della stessa e la necessità di sostegno del Top Management per la sua diffusione. Il modello non possiede tuttavia validazione empirica. Ancora più recentemente, Kamitri et al. (2017) pubblicano i risultati di una Review sistematica della letteratura apparsa tra il 2004 e il 2014 e catturata da database medici (Medline, Cinahl and Health Source: nursing/academic edition); sono analizzati e discussi

\footnotetext{
${ }^{2} \mathrm{Si}$ tratta in particolare del Knowledge Management Conceptualization in Healthcare (KMCH) dell'Università di Victoria (School of Health Information Science) (Lau, 2004), del Total Knowledge Management in healthcare (TKMh) proposto gruppo di ricerca del KM in sanità del Coventry University, UK (Bali et al. 2009) e del Knowledge Management Infrastructure in Healthcare sviluppato dalla scuola di Business dell’Università Australiana RMIT (Wickramasinghe, 2010).
} 
7 temi centrali: (1) la percezione del KM, (2) la generazione di conoscenza, (3) la sua disseminazione, (4) la cooperazione, (5) gli strumenti di trasferimento della conoscenza; (6) la leadership; (7) le barriere organizzative. Dall'analisi emergono numerose difficoltà nell'implementazione del $K M$ in sanità (e sulle quali intervenire), riconducibili in particolare ai limiti di tempo e di competenza. È dunque enfatizzata l'importanza del management e dei relativi strumenti di supporto nonché un atteggiamento aziendale collaborativo, intesi quali broker della conoscenza. Sugli stessi temi si pone anche il lavoro di Bordoloi e Islam (2012), che tuttavia arriva alla mera postulazione di ipotesi di ricerca senza condurre analisi esperienziali né revisioni sistematiche della letteratura. Non mancano i casi di studio: dal lavoro ormai datato di Dwivedi et al. (2003) relativo a 27 realtà inglesi, in cui si riscontrava l'assenza di strumenti operativi in grado di trasformare le idee concettuali del $K M$ in effettivi piani strategici, a quello di Yamazaki e Umemoto (2010), che attraverso l'analisi di 2 casi di studio giapponesi evidenziano la complessa applicazione di noti paradigmi di KM al contesto sanitario (Nonaka \& Takeuchi, 1995) sino al contributo di Griffith et al. (2012) che attraverso l'analisi di nove casi di successo da fonte secondaria (content analysis delle candidature al premio Baldrige) concludono sull'importanza di misurare, confrontarsi e migliorare le organizzazioni attraverso la creazione, l'impiego, la conservazione e il trasferimento della conoscenza.

Tabella 2 - Ambito di analisi e approccio metodologico

\begin{tabular}{l|l|l|l}
\hline & Conceptual paper & Literature Review & Case studies \\
\hline $\begin{array}{l}\text { Sviluppo/affinamento } \\
\text { di framework }\end{array}$ & Sabeeh et al. (2016) & $\begin{array}{l}\text { Ferlie et al. (2012) } \\
\text { Bordoloi e Islam (2012) } \\
\text { Karamitri et al. (2017) }\end{array}$ & $\begin{array}{l}\text { Dwivedi et al. (2003): MCS - MIX* } \\
\text { Yamazaki e Umemoto (2010): PCS }-Q^{*} \\
\text { Griffith et al. (2013): NCS - QUAL* }\end{array}$ \\
\hline Driver del valore & Macdonald (2003) & Delgosha et al. (2013) & $\begin{array}{l}\text { Wu e Hu (2012): OV - QNT* } \\
\text { Thim e Weber (2012): CSS }-Q^{*}\end{array}$ \\
\hline Barriere organizzative
\end{tabular}

* CSS (caso di studio singolo); PCS (pochi casi di studio: sino a 10); NCS (numerosi casi di studio: sopra i 10); QUAL (Qualitativo); QNT (Quantitativo); MIX (Qualitativo e Quantitativo); OV (orientato alle variabili); ND (non disponibile) Fonte: ns elaborazione

Altro pilastro della ricerca aziendale (e del $K M$ in particolare) è rappresentato dai driver del valore, ovvero dai processi aziendali o dalle risorse che meglio degli altri consentono di allargare la forbice tra la monetizzazione dei beni (o dei servizi) venduti (o erogati) e la monetizzazione dei fattori produttivi impiegati nei processi aziendali tipici. Macdonald (2002), a tal proposito, individua numerosi indicatori prospettici (in ottica dunque feed-forward) volti a misurare 7 importanti dimensioni del valore: (1) reclutamento e conservazione di risorse umane appropriate, (2) sviluppo di competenze e abilità strategiche, (3) generazione di un clima favorevole all'apprendimento, (4) valorizzazione dell'impegno e dello spirito di iniziativa, (5) individuazione e impiego dell'evidenza empirica come parte integrante della pratica e del processo decisionale, (6) governo e impiego delle informazioni, (7) trasferimento interno ed esterno di conoscenza. Più recentemente Delgosha et al. (2012) conducono una revisione mirata della letteratura attraverso la quale propongono il concetto di gestione della conoscenza del paziente (Patient Knowledge Management), intesa quale insieme di valori, bisogni e preferenze degli stessi pazienti da cui derivare pratiche di co-produzione (Nabatchi et al., 2017; Brandsen e Honingh 2016) sperimentare nuove tecniche di trattamento e pervenire a riduzioni di costo. Né Macdonald né Delgosha et al. supportano tuttavia le proprie proposte con materiale empirico di rilievo. Casi concreti di studio sono invece proposti da Thim e Weber (2012) e da Wu e Hu (2012). I primi sviluppano un singolo caso di studio tedesco, mostrando la differenza di percezione dei driver del valore esistente tra medici, manager e professioni infermieristiche; concludono rappresentando la necessità di pervenire a misurazioni oggettive, pur preservando la ricchezza delle singole prospettive e sensibilità. Wu e Hu lavorano invece su un modello quantitativo orientato alle variabili (che testano su 144 centri medici/ospedali) confermando empiricamente, tra le altre, la relazione positiva tra le competenze nella gestione della conoscenza, da una parte, e le performance (economiche e qualitative) dall'altra.

Vari contributi si soffermano infine sui fattori e sulle condizioni organizzative che favoriscono o ostacolano lo sviluppo di strategie orientate alla gestione della conoscenza. È il caso di Booth (2000), che in una fase ancora primordiale degli studi di Healthcare Knowledge Management, intravedeva uno spreco di risorse negli investimenti in tecnologia ove non accompagnati dallo sviluppo di competenze e tecniche di gestione delle 
informazioni, individuabile nel Content Management, nella profilazione degli utenti e nell'appropriatezza delle ricerche online. Più recentemente, sempre sul rapporto tra tecnologia e $K M$, Williams e Dickinson (2010) hanno effettuato una review della letteratura dalla quale hanno desunto la difficoltà e l'incertezza di effettuare previsioni sul successo degli investimenti in tecnologia; i comportamenti, infatti, non sempre appaiono razionali mentre i cambiamenti di contesto sono frequenti e discontinui. Kothari et al. (2011) e Oborn et al. (2013) concentrano invece le proprie analisi sui fattori di stimolo delle pratiche di $K M$, i primi enfatizzando il ruolo della cultura e delle strutture organizzative, l'importanza del sostegno ai manager interpreti del cambiamento, la necessità di chiare strategie orientate alla gestione della conoscenza e l'opportunità di adattare i sistemi di valutazione delle performance; i secondi presentando l'esperienza di specifiche realtà nate con l'obiettivo di supportare il cambiamento organizzativo; è il caso di CLAHRCs (Collaboration for Leadership in Applied Health Research and Care), di HIECs (Health Innovation and Education Cluster) o di AHSNs (Academic Health Science Networks). Come per le precedenti aree tematiche anche qui non mancano i casi aziendali. Persson e Stirna (2007) analizzano in profondità un singolo caso svedese intravedendo, ancor prima delle sistematizzazioni teoriche, l'importanza di supportare il cambiamento con iniziative ad hoc quali lo sviluppo partecipato e contestualizzato dei progetti di $K M$, la previsione di un supporto organizzativo e tecnico espressamente dedicato al cambiamento, la presenza di esperienze pilota e di strumenti di verifica dei risultati. Più recenti sono i lavori Cruz e Ferreira (2016) e di Bresnen et al. (2017). I primi mostrano che l'apertura verso il $K M$ quale fattore strategico dipende dal tipo di organizzazione e di attività: più sentita nelle organizzazioni ospedaliere complesse (ove generalmente le dimensioni sono medio-grandi e il grado di tecnologia e di specializzazione è elevato), l'importanza di gestire le conoscenze è meno avvertita tra gli operatori di strutture che erogano cure primarie. I secondi si concentrano su 3 casi di studio inglesi e concludono sull'importanza di superare il pensiero prevalentemente dicotomico dei clinici, recuperando la pienezza di ruolo dei manager, anche attraverso la leva premiale, e valorizzando il ruolo dei cosiddetti ibrid manager (coordinatori infermieristici, direttori di dipartimento medico, dirigenti di laboratorio e farmacia etc).

Tabella 3 - Fattori strategici di successo per ambito di analisi

\begin{tabular}{|c|c|c|c|}
\hline Fattore strategico di successo & Framework & Driver del valore & Barriere organizzative \\
\hline $\begin{array}{l}\text { Leadership - in particolare degli ibrid } \\
\text { manager e delle direzioni } \\
\text { generali/amministrative - loro supporto } \\
\text { con deleghe reali e incentivi economici }\end{array}$ & $\begin{array}{l}\text { Cruz e Ferreira (2016) } \\
\text { Sabeeh et al. (2016) } \\
\text { Karamitri et al. (2017) }\end{array}$ & $\begin{array}{l}\text { Macdonald (2003) } \\
\text { Thim e Weber (2012) }\end{array}$ & $\begin{array}{l}\text { Williams and Dickinson (2010) } \\
\text { Kothari et al. (2011) } \\
\text { Cruz e Ferreira (2016) } \\
\text { Brensen et al. (2017) }\end{array}$ \\
\hline $\begin{array}{l}\text { Cultura organizzativa - presenza di } \\
\text { figure dedicate al KM e percezione da } \\
\text { parte dei operatori del ruolo del KM }\end{array}$ & $\begin{array}{l}\text { Cruz e Ferreira (2016) } \\
\text { Sabeeh et al. (2016) } \\
\text { Karamitri et al. (2017) }\end{array}$ & $\begin{array}{l}\text { Macdonald (2003) } \\
\text { Thim e Weber (2012) }\end{array}$ & $\begin{array}{l}\text { Persson e Stirna (2007) } \\
\text { Williams and Dickinson (2010) } \\
\text { Kothari et al. (2011) } \\
\text { Cruz e Ferreira (2016) }\end{array}$ \\
\hline $\begin{array}{l}\text { Processi di sintesi/esplicitazione di } \\
\text { conoscenza }\end{array}$ & $\begin{array}{l}\text { Dwivedi et al. (2003) } \\
\text { Wu e Hu (2012) } \\
\text { Bordoloi e Islam (2012) } \\
\text { Sabeeh et al. (2016) } \\
\text { Karamitri et al. (2017) }\end{array}$ & $\begin{array}{l}\text { Macdonald (2003) } \\
\text { Wu e Hu (2012) } \\
\text { Griffith et al. (2013) }\end{array}$ & $\begin{array}{l}\text { Booth (2000) } \\
\text { Persson e Stirna (2007) } \\
\text { Williams and Dickinson (2010) }\end{array}$ \\
\hline $\begin{array}{l}\text { Processi di } \\
\text { disseminazione/trasferimento di } \\
\text { conoscenza }\end{array}$ & $\begin{array}{l}\text { Dwivedi et al. (2003) } \\
\text { Wu e Hu (2012) } \\
\text { Sabeeh et al. (2016) } \\
\text { Karamitri et al. (2017) }\end{array}$ & $\begin{array}{l}\text { Wu e Hu (2012) } \\
\text { Griffith et al. (2013) }\end{array}$ & $\begin{array}{l}\text { Booth (2000) } \\
\text { Persson e Stirna (2007) } \\
\text { Oborn et al. (2013) }\end{array}$ \\
\hline $\begin{array}{l}\text { Processi di } \\
\text { cooperazione/socializzazione di } \\
\text { conoscenze (tacite) }\end{array}$ & $\begin{array}{l}\text { Dwivedi et al. (2003) } \\
\text { Yamazaki e Umemoto } \\
(2010) \\
\text { Karamitri et al. (2017) }\end{array}$ & $\begin{array}{l}\text { Macdonald (2003) } \\
\text { Delgosha et al. (2013) }\end{array}$ & $\begin{array}{l}\text { Booth (2000) } \\
\text { Williams and Dickinson (2010) } \\
\text { Oborn et al. (2013) }\end{array}$ \\
\hline $\begin{array}{l}\text { Strumenti di trasferimento della } \\
\text { conoscenza - agenzie ad hoc, presenza } \\
\text { di un piano strategico di KM, strumenti } \\
\text { tecnologici e informativi a supporto delle } \\
\text { decisioni, formazione }\end{array}$ & $\begin{array}{l}\text { Dwivedi et al. (2003) } \\
\text { Wu e Hu (2012) } \\
\text { Sabeeh et al. (2016) } \\
\text { Karamitri et al. (2017) }\end{array}$ & $\begin{array}{l}\text { Wu e Hu (2012) } \\
\text { Griffith et al. (2013) }\end{array}$ & $\begin{array}{l}\text { Persson e Stirna (2007) } \\
\text { Williams and Dickinson (2010) } \\
\text { Kothari et al. (2011) } \\
\text { Oborn et al. (2013) }\end{array}$ \\
\hline
\end{tabular}

Fonte: ns elaborazione

Al di là della specifica area di indagine e del metodo di ricerca impiegato è interessante notare che $\mathrm{i}$ ricercatori convergono sulla centralità alcuni fattori strategici di successo, che appaiono dunque trasversali e capaci di attenuare le barriere organizzative all'introduzione delle pratiche di KM e, in seconda battuta, di contribuire alla generazione di valore. Si va dalle componenti soft degli stili di leadership e più in generale della cultura del cambiamento supportate da logiche premiali e da risorse dedicate, al governo dei processi di sintesi, trasferimento e socializzazione delle competenze, sino agli strumenti tecnici di supporto al management della conoscenza che comprendono l'elaborazione di piani strategici ad hoc, la costituzione di 
agenzie formative, la disponibilità di sistemi informativi a supporto delle decisioni (cfr., per i dettagli, tabella 3).

Ciononostante, a differenza di quanto ci si sarebbe attesi, indipendentemente dalla metodologia o dall'area tematica affrontata, è scarsamente approfondita la relazione tra le misure di valore, il contesto organizzativo e la tipicità dei processi sottesi. Complessivamente sono stati individuati 3 framework che tuttavia o debbono ancora essere oggetto di validazione empirica (Sabeeh et al., 2016; Cruz et al., 2016) o non entrano nel merito dei processi che generano leva competitiva (Wu e Hu, 2012).

\section{Conclusioni e prospettive future di analisi}

Alla luce dei risultati della ricerca condotta (cfr., supra, paragrafo 5) e stanti i limiti connessi alle scelte metodologiche effettuate (cfr., supra, paragrafo 4), si intende adesso rispondere ai tre quesiti che hanno motivato il presente lavoro.

Un primo interrogativo era relativo alla presenza di studi di Healthcare Knowledge Management che affrontassero specificatamente argomenti di management (innovazione di prodotto, organizzazione e gestione per processi, dinamiche del valore, ad esempio), anche in rapporto a quelli incentrati sulla gestione delle informazioni in ambito clinico, tecnologico o informatico. L'analisi effettuata ha svelato una presenza robusta di contributi incentrati sulla gestione delle informazioni di tipo squisitamente sanitario (percorsi dei pazienti affetti da specifica patologia) o tecnologico (soluzioni Hi-Tech di interfaccia delle attrezzature elettromedicali, di governo dell'ingegneria clinica e della semiotica/semantica informatica). È tuttavia stata osservata anche una presenza quantitativamente significativa di saggi a taglio manageriale, che rappresenta circa un terzo dei contributi intercettati dai database consultati; a dimostrare che la gestione della conoscenza rappresenta un tema profondamente interdisciplinare, ove uno spazio significativo è occupato dal management.

In base a questa ricognizione, ci si è domandati quali degli studi incentrati sui temi propri del management avessero sviluppato framework distintivi (avuto dunque cura delle caratteristiche tipiche delle aziende analizzate - strutture, processi e ambiente economico/istituzionale di riferimento) e, in subordine, se tali schemi interpretativi fossero stati testati. L'evidenza raccolta presenta numerosi casi di studio, varie analisi della letteratura e alcuni conceptual paper che appaiono incentrati sulle barriere o i fattori di stimolo organizzativo al cambiamento mentre l'elaborazione di chiavi interpretative specifiche per le aziende sanitarie risulta modesta; si tratta in particolare di 3 lavori, 2 dei quali interessanti perché recenti e inclusivi delle conclusioni di modelli precedenti (Sabeeh et al., 2016; Cruz e Ferreira, 2016), uno perché testato su un campione numeroso di strutture sanitarie (Wu e Hu, 2012). Manca tuttavia un'approfondita analisi delle caratteristiche di sistema e dei processi tipici delle aziende che motiva l'adozione delle variabili considerate come pure è assente (tranne in Wu e $\mathrm{Hu}, 2012$ ) la validazione empirica delle tesi proposte.

$\mathrm{Ci}$ si è chiesti, infine, se la letteratura manageriale internazionale avesse opportunamente esplicitato le dimensioni del valore, sia con riferimento ai suoi driver che con riguardo alla sua misurazione. Qui, la ricerca non fornisce evidenza di contributi significativi; il tema del valore pubblico sembra ancora ancorato ad elaborazioni squisitamente teoriche che non hanno ancora trovato nel $K M$ la prospettiva di interpretazione dei fenomeni reali e che, tantomeno, hanno elaborato modelli di business utili alla definizione di scelte strategiche e alle relative misure di riferimento.

Alla luce di tutto quanto sopra sembra di poter concludere che l'attuale produzione scientifica di Healthcare Knowledge Management non presenta elementi di grande originalità ma che, piuttosto, proponga degli adattamenti perlopiù acritici di paradigmi sviluppati con successo nelle imprese. In effetti, come illustrato pocanzi, i lavori ad oggi pubblicati pongono scarsa attenzione sui processi tipici e sulle caratteristiche strutturali/ambientali delle organizzazioni sanitarie. Benché le analisi di casi aziendali non manchino, probabilmente proprio a causa della scarsa contestualizzazione esse appaiono incentrate su aspetti di nicchia, dimenticando di esplorare la dinamica del valore aziendale.

Per gli studiosi si apre dunque lo spazio e l'opportunità di elaborare e testare Business Model coerenti con la dinamica dei sistemi sanitari e con gli assetti delle relative aziende. Più in dettaglio sembra opportuno ipotizzare, quindi verificare, quali siano i driver specifici del valore nelle aziende sanitarie, partendo da una robusta analisi delle esperienze disponibili sul tema e sviluppando framework ad hoc da operazionalizzare, quindi sperimentare mediante l'analisi di selezionati casi di studio. Tali analisi, date le profonde differenze che contraddistinguono le varie realtà organizzative, dovrebbero essere svolte distintamente, indipendentemente dalle denominazioni che assumono nei vari contesti, almeno per ospedali pubblici, case di cura convenzionate, case di riposo e cure primarie. Interessante, a tal proposito, la prospettiva del Valore Pubblico Prodotto per 
come formulata dalla dottrina economico aziendale italiana, che peraltro ben si pone in linea con gli apporti manageriali anglosassoni recuperando tuttavia la visione olistica dell'azienda. Con stretto riferimento alle aziende composte pubbliche, essa riconduce il soddisfacimento del principio di economicità a cinque equilibri fondamentali: oltre a quelli reddituale e monetario, propri di ogni classe di azienda, a quelli distintivi di equilibrio di consumo, tributario e di consenso (Garlatti, 2017).

\section{Riferimenti bibliografici}

Aa Vv. (2012). La gestione dei sistemi sanitari negli Stati membri dell'UE. Il ruolo degli enti locali e regionali. Bruxelles: Comitato europeo delle Regioni.

Alford J., Douglas S., Geuijen K. \& Paul 't Hart (2016). Ventures in public value management: introduction to the symposium, Public Management Review, 19 (5): 589-604. DOI: 10.1080/14719037.2016.1192160.

Booth A. (2000). Knowledge Management in the NHS: half full or half empty? VINE: The journal of information and knowledge management systems, 30 (4): 19-23. DOI: 10.1108/eb040771.

Bordoloi P., Islam N. (2012), Knowledge Management Practices and Healthcare Delivery: A Contingency Framework, The Electronic Journal of Knowledge Management, 10 (2): 110-120.

Borgonovi E., Meneguzzo M. (1990). Opportunities for the development of competitive elements in the Italian healthcare system: public competition, private health insurances, non profit. In: Caspaire A.F., Hermans H.E., Paelink J.H.P. (eds). Competitive healthcare in Europe. Future prospects, Aldershot: Dartmouth.

Brandsen T., Honingh M. (2016). Distinguishing Different Types of Coproduction: A Conceptual Analysis Based on the Classical Definitions, Public Administration Review 76 (3): 427-35. DOI: 10.1111/puar.12465.

Bresnen M., Hodgson D., Bailey S., Hyde P., Hassard J. (2017). Mobilizing management knowledge in healthcare: Institutional imperatives and professional and organizational mediating effects, Management Learning, 48 (5): 597-614. DOI: $10.1177 / 1350507617718257$.

Cacace M., Ettelt S., Mays N., Nolte E. (2013). Assessing quality in cross-country comparisons of health systems and policies: Towards a set of generic quality criteria, Health Policy, 112 (1-2): 156-162. DOI: 10.1016/j.healthpol.2013.03.020.

Cegarra-Navarro J.G., Sánchez A.L.G., Cegarra J.L.M (2012). Creating patient e-knowledge for patients through telemedicine technologies, Knowledge Management Research and Practice, 10 (2): 153-163. DOI: 10.1057/kmrp.2011.47.

Cruz S.G., Ferreira M.M. (2016). Knowledge management in Portuguese healthcare institutions, Revista brasileira de enfermagem, 69 (3): 492-499. DOI: 10.1590/0034-7167.2016690311i.

Cruz-Cunha M.M., Miranda I., Lopes N., Simoes R. (2013). An e-marketplace of healthcare and social care services: the perceived interest, The Learning Organization, 20 (6): 406-418. DOI: 10.1108/TLO-10-2013-0055.

Delgosha M.S., Ojaki A.A., Farhadi H. (2013). The business values of patient knowledge management (PKM) in the healthcare industry, International Journal of Healthcare Information Systems and Informatics, 8 (2): 70-79. DOI: 10.4018/jhisi.2013040106.

Donahue J. D., Moore M. H. (eds) (2012). Ports in a Storm: Public Management in a Turbulent World. Washington D.C.: Brookings Institution Press.

Dwivedi A.N., Bali R.K., Naguib R.N.G. (2003). Organization Current Knowledge Design (OCKD): A Knowledge Management Framework for Healthcare Institutions. In: Proceedings of Annual International Conference of the IEEE Engineering in Medicine and Biology.

Ferlie E., Crilly T., Jashapara A., Peckham A. (2012). Knowledge mobilisation in healthcare: A critical review of health sector and generic management literature, Social Science and Medicine, 74 (8): 1297-1304. DOI: 10.1016/j.socscimed.2011.11.042.

Griffith J.R., Fear K.M., Lammers E., Banaszak-Holl J., Zheng K. (2013). A positive deviance perspective on hospital knowledge management: Analysis of baldrige award recipients 2002-2008, Journal of Healthcare Management, 58 (3): 187-203.

Kaplan S. (2011). Business Models Aren't Just For Business, Harvard Business Review, April.

Karamitri I., Talias M.A., Bellali T. (2017). Knowledge management practices in healthcare settings: a systematic review, International Journal of Health Planning and Management, 32 (1): 4-18. DOI: 10.1002/hpm.2303.

Kothari A., Hovanec N., Hastie R., Sibbald S. (2011). Lessons from the business sector for successful knowledge management in health care: A systematic review, BMC Health Services Research, 173: 1-11. DOI: 10.1186/1472-6963-11-173.

Levy A., Sobolev B. (eds) (2016). Comparative effectiveness research in health services. New York: Springer References.

Garlatti A., Massaro M., Dumay J. (2015). Knowledge Management within the public sector: A structured literature review, Journal of Knowledge Management, 19 (3): 530-558. DOI: 10.1108/JKM-11-2014-0466.

Garlatti A. (2017). L'economicità nelle aziende composte pubbliche. I 5 equilibri fondamentali. In: Cescon F., Garlatti A. (a cura di). Economia Aziendale. Casi e Testi - Terza edizione, Milano: Wolters Kluver.

Lombrano A. (2018). La comparazione dei sistemi sanitari. Uniformità, eterogeneità e risultati, Rivista Italiana di Ragioneria ed Economia Aziendale, (2): Forthcoming.

Longo F., Ricci A. (2017). La sanità italiana a metà del guado: quali direzioni? In: Cergas - SDA Bocconi (a cura di), Rapporto OASI 2017. Milano: Egea.

Mabery M.J., Gibbs-Scharf L., Bara D. (2013). Communities of practice foster collaboration across public health, Journal of Knowledge Management, 17 (2): 226-236. DOI: 10.1108/13673271311315187.

Macdonald M. (2003). Knowledge Management in Healthcare: What Does It Involve? How Is It Measured? Healthcare Management Forum, 16 (3): 7-11. DOI: 10.1016/S0840-4704(10)60225-6.

Masini C. (1970). Lavoro e risparmio. Torino; UTET.

Massaro M., Dumay J., Guthrie J. (2016). On the shoulders of giants: undertaking a structured literature review in accounting, Accounting, Auditing \& Accountability Journal, 29 (5): 767-801. DOI: 10.1108/AAAJ-01-2015-1939.

Moore M. (1995). Creating Public Value: Strategic Management in Government. Cambridge, MA: Harvard University Press.

Moore M.H. (2013). Recognizing Public Value. Boston: Harvard University Press. 
Morrell K. (2009). Governance and the Public Good, Public Administration, 87 (3): 538-56. DOI: 10.1111/j.1467-9299.2009.01756.x. Nabatchi T., Sancino A, Sicilia M. (2017). Varieties of Participation in Public Services: The Who, When, and What of Coproduction, Public Administration Review, 77 (5): 766-76. DOI: 10.1111/puar.12765.

Oborn E., Barrett M., Racko G. (2013). Knowledge translation in healthcare: Incorporating theories of learning and knowledge from the management literature, Journal of Health Organization and Management, 27 (4): 412-431. DOI: 10.1108/JHOM-01-20120004.

Papnicolas I., Smith P.C. (eds) (2013). Health System Performance Comparison. An agenda for policy, information and research. Maidenhead: McGraw-Hill Education.

Persson A., Stirna J. (2007). Making it happen and making it stick-a successful case of implementing a knowledge management system in a healthcare organization. In: Vv. Aa. Proceedings of the 15th European Conference on Information Systems, ECIS.

Riccucci N. M. (1995). Unsung Heroes: Federal Execucrats Making a Difference. Washington, DC: Georgetown University Press.

Sabeeh Z.A., Mustapha S.M.F.D.S., Mohamad R. (2016). A Review of Three Models for Knowledge Management in Healthcare Organizations, In: Vv. Aa. Proceedings of Knowledge Management International Conference, Chiang Mai (Thailand): KMICe.

Snowdon A., Chen J. (2011). Strengthening health systems through innovation: lessons learned. Ivey International Centre for Health Promotion, London: Western University.

Thim C., Weber N. (2012). Management of tacit knowledge to overcome learning barriers between professions. In: Proceedings of the European Conference on Knowledge Management, ECKM.

Varabyova Y., Schreyögg J. (2013). International comparisons of the technical efficiency of the hospital sector: Panel data analysis of OECD countries using parametric and non-parametric approaches, Health Policy, 112 (1-2): 70-79. DOI: 10.1016/j.healthpol.2013.03.003.

Williams L., Dickinson H. (2010). Can knowledge management enhance technology adoption in healthcare? A review of the literature. Evidence and Policy, 6 (3): 309-331. DOI: 10.1332/174426410X524811.

Wu I.L., Hu Y.P. (2012). Examining knowledge management enabled performance for hospital professionals: A dynamic capability view and the mediating role of process capability, Journal of the Association of Information Systems, 13 (12): 976-999.

Yamazaki T., Umemoto K. (2010). Knowledge management of healthcare by clinical-pathways, Journal of Information and Knowledge Management, 9 (2): 119-125. DOI: 10.1142/S0219649210002577. 